\title{
Asymptotics of the hitting probability for a small sphere and a two dimensional Brownian motion with discontinuous anisotropic drift
}

\author{
PETER GRANDITS \\ Institut für Stochastik und Wirtschaftsmathematik, TU Wien, Wiedner Hauptstraße 8-10, A-1040 Wien, Austria. \\ E-mail: pgrand@fam.tuwien.ac.at
}

We provide an approximation of the hitting probability for a small sphere for the following two dimensional process: In $x$-direction it is just a Brownian motion with positive constant drift, whereas in $y$-direction the process $Y_{t}$ is a Brownian motion with drift given by a negative constant times the sign of $Y_{t}$. This process can be seen as the solution of a certain stochastic optimal control problem. It turns out that the approximating function can be expressed as the sum of a term involving a modified Bessel function and an ordinary Lebesgue integral.

Keywords: Discontinuous drift; hitting probabilities; optimal control problem; two-dimensional Brownian motion

\section{Introduction}

The calculation respectively the determination of the asymptotic behavior for hitting probabilities under various assumption is a classical topic in probability theory. For example, there exists a vast literature in ruin theory, where the aim is, to estimate the probability that a certain stochastic process hits the negative real numbers. The most classical result in this direction stems from F. Lundberg [12], where the underlying process is a compound Poisson process, and it is shown that, under certain assumptions, the ruin probability can be estimated by an exponential function, depending on the initial value of the process. An overview of this topic can be found in the monograph [1].

Another problem would be to consider the multidimensional situation, and to ask for large deviation results for the probability that a process hits a certain set, see [4] and the references therein.

In our paper, we want to consider the hitting probabilities for small spheres. It is well known that for a two-dimensional Brownian motion this probability is equal to one, since the process is recurrent for discs, and for higher dimension there exists a well known formula for this probability, see, for example, [9]. The problem for a Brownian motion with constant drift in dimension $d \geq 2$ is considered in [17], where a Laplace-Gegenbauer transform for hitting time and place is calculated, and as corollary an infinite series for the hitting probability is given (see [16] for the case without drift).

Here we want to consider the two dimensional situation only, and we shall assume that the process in $x$-direction is given by a Brownian motion with positive constant drift and volatility, whereas the process in $y$-direction, say $Y_{t}$, is a Brownian motion with drift given by a negative constant times $\operatorname{sgn}\left(Y_{t}\right)$. It is well known, see [6], that $\left|Y_{t}\right|$ is a representation of reflected Brownian motion with negative drift.

Moreover, we shall show in Section 5 that this two-dimensional process solves the following stochastic optimal control problem. Consider a particle in a streaming fluid, where the controller can influence only the movement perpendicular to the streaming. The aim is to maximize the hitting probability for some small disc. Let us note that a similar problem in dimension one has been considered in [2], problem 1, where the solution process is of a similar type (see also [9], Section 6.5). 
The paper, which is closest to ours is [13] (see also [10] for the problem in dimension greater than 2). There an asymptotic result for the hitting probability is derived for certain diffusion processes. The result of [13] assumes Hölder continuous coefficients, which is clearly not the case in our model. Our main result will be an approximation of the hitting probability, which can be expressed as the sum of a term involving a modified Bessel function and an ordinary Lebesgue integral. Let us close this introduction by mentioning a paper on hitting probabilities for a uni-modal isotropic Levy process [7].

The schedule of the paper will be the following. In Section 2, we give some preliminary notions, and we formulate and prove our main result. In Section 3, we show that our process solves the stochastic control problem mentioned above, and finally, in Section 4, we consider some degenerate cases, where one of the volatilities is set to zero. Two numerical examples are also provided in this section.

\section{The model, preliminary definitions and the main result}

We start with the introduction of the basic model. Let $Z_{t}:=\left(X_{t}, Y_{t}\right)$ given by

$$
\begin{aligned}
& X_{t}=x+\mu_{1} t+\sigma_{1} W_{t}^{(1)}, \\
& Y_{t}=y-\mu_{2} \int_{0}^{t} \operatorname{sgn}\left(Y_{s}\right) d s+\sigma_{2} W_{t}^{(2)},
\end{aligned}
$$

where $\mu_{1}, \mu_{2}, \sigma_{1}, \sigma_{2}$ are positive constants, and $W^{(1)}$ and $W^{(2)}$ are independent Brownian motions. We note that the second SDE has, by a result of Zvonkin [18], a unique strong solution.

Furthermore, let $K:=B(0, \delta)$ a circle with radius $\delta$ and the origin as center, and define

$$
\tau:=\inf \left\{t>0 \mid Z_{t} \in K\right\}
$$

Our aim is to find an approximation of the quantity $\mathbf{P}(\tau<\infty)$, for small $\delta$ and $(x, y)$ fulfilling $x^{2}+$ $y^{2} \geq \delta^{2}$

As usual, we write $f(x, y, \delta) \sim g(x, y, \delta)$, if we have

$$
\lim _{\delta \rightarrow 0} \frac{f(x, y, \delta)}{g(x, y, \delta)}=1 .
$$

We state now the main result of the paper

Theorem 2.1. We have for the hitting probability $\mathbf{P}(\tau<\infty)$ the asymptotic relation

$$
\mathbf{P}(\tau<\infty) \sim \pi u\left(\frac{x}{\sigma_{1}}, \frac{y}{\sigma_{2}} ; \frac{\mu_{1}}{\sigma_{1}}, \frac{\mu_{2}}{\sigma_{2}}\right) \frac{1}{(-\ln \delta)},
$$

uniformly for $\sqrt{x^{2}+y^{2}} \geq \delta \frac{\max \left(\sigma_{1}, \sigma_{2}\right)}{\min \left(\sigma_{1}, \sigma_{2}\right)}$, where $u\left(x, y ; \mu_{1}, \mu_{2}\right)$ is given by

$$
\begin{aligned}
u\left(x, y ; \mu_{1}, \mu_{2}\right)= & \frac{e^{-\mu_{1} x+\mu_{2}|y|}}{\pi} K_{0}\left(\sqrt{x^{2}+y^{2}} \sqrt{\mu_{1}^{2}+\mu_{2}^{2}}\right) \\
& +\int_{0}^{\infty} \frac{\mu_{2}}{2 \sqrt{2 \pi t}} \exp \left(-\frac{x^{2}}{2 t}-\frac{\mu_{1}^{2} t}{2}-\mu_{1} x\right) \operatorname{erfc}\left(\frac{|y|-\mu_{2} t}{\sqrt{2 t}}\right) d t
\end{aligned}
$$


Proof. We start with

Case 1: $\sigma_{1}=\sigma_{2}=1$.

The transition density of $X_{t}$ is equal to

$$
p_{t}^{X}\left(x_{1}, x_{2}\right)=\frac{1}{\sqrt{2 \pi t}} \exp \left(-\frac{\left(x_{2}-x_{1}-\mu_{1} t\right)^{2}}{2 t}\right) .
$$

The transition density of $Y_{t}$ can be found in Section 15, Appendix 1 of [3] or [9], (6.5.14); evaluated at $y_{2}=0$ it is given by

$$
p_{t}^{Y}\left(y_{1}, y_{2}=0\right)=\frac{1}{\sqrt{2 \pi t}} \exp \left(-\frac{\left(\left|y_{1}\right|-\mu_{2} t\right)^{2}}{2 t}\right)+\frac{\mu_{2}}{2} \operatorname{erfc}\left(\frac{\left|y_{1}\right|-\mu_{2} t}{\sqrt{2 t}}\right) .
$$

Since $X_{t}$ and $Y_{t}$ are independent, the transition density of $\left(X_{t}, Y_{t}\right)$ is $p_{t}\left(\left(x_{1}, y_{1}\right),\left(x_{2}, y_{2}\right)\right)=p_{t}^{X}\left(x_{1}\right.$, $\left.x_{2}\right) p_{t}^{Y}\left(y_{1}, y_{2}\right)$. The potential kernel (see, e.g., [14], ex. III.2.29) with pole at $(0,0)$ is thus equal to

$$
u(x, y)=\int_{0}^{\infty} p_{t}((x, y),(0,0)) d t=\int_{0}^{\infty} p_{t}^{X}(x, 0) p_{t}^{Y}(y, 0) d t
$$

where we have written now $x$ for $x_{1}$ and $y$ for $y_{1}$, for convenience.

Using the explicit expressions above, we find that

$$
\begin{aligned}
u(x, y)= & \int_{0}^{\infty} \frac{1}{2 \pi t} \exp \left(-\left(x^{2}+y^{2}\right) /(2 t)-\left(\mu_{1}^{2}+\mu_{2}^{2}\right) t / 2-\mu_{1} x+\mu_{2}|y|\right) d t \\
& +\int_{0}^{\infty} \frac{\mu_{2}}{2 \sqrt{2 \pi t}} \exp \left(-x^{2} /(2 t)-\mu_{1}^{2} t / 2-\mu_{1} x\right) \operatorname{erfc}\left(\left(|y|-\mu_{2} t\right) / \sqrt{2 t}\right) d t
\end{aligned}
$$

The first integral is simply

$$
\frac{\exp \left(-\mu_{1} x+\mu_{2}|y|\right)}{\pi} K_{0}\left(\sqrt{x^{2}+y^{2}} \sqrt{\mu_{1}^{2}+\mu_{2}^{2}}\right),
$$

where $K_{0}$ denotes a modified Bessel function of the second kind, and as $(x, y) \rightarrow 0$, it is equal to

$$
-\frac{1}{\pi} \ln \sqrt{x^{2}+y^{2}}+O(1)
$$

Using the estimate $0 \leq \operatorname{erfc}(s) \leq 2$, we find that the other integral is non-negative and bounded by

$$
\int_{0}^{\infty} \frac{\mu_{2}}{\sqrt{2 \pi t}} \exp \left(-x^{2} /(2 t)-\mu_{1}^{2} t / 2-\mu_{1} x\right) d t=\frac{\mu_{2}}{\mu_{1}} e^{-\mu_{1}(|x|+x)} \leq \frac{\mu_{2}}{\mu_{1}} .
$$

It follows that

$$
u(x, y)=-\frac{1}{\pi} \ln \sqrt{x^{2}+y^{2}}+O(1)
$$

as $(x, y) \rightarrow 0$, that is,

$$
-C \leq u(x, y)+\frac{1}{\pi} \ln \sqrt{x^{2}+y^{2}} \leq C,
$$

for $x^{2}+y^{2} \leq 1$ and some positive constant $C$, depending on the $\mu_{i}$. 
By the mean-value property for the potential kernel, we have

$$
u(x, y)=\mathbf{E}^{(x, y)}\left[\mathbf{1}_{\{\tau<\infty\}} u\left(X_{\tau}, Y_{\tau}\right)\right]
$$

This is shown in the Appendix. Thus,

$$
\frac{u(x, y)}{\sup _{\partial K} u} \leq \mathbf{P}^{(x, y)}(\tau<\infty) \leq \frac{u(x, y)}{\inf _{\partial K} u} .
$$

Now, we find

$$
\begin{aligned}
\sup _{\partial K} u(x, y) & =\sup _{\partial K}\left(u(x, y)+\frac{1}{\pi} \ln \sqrt{x^{2}+y^{2}}-\frac{1}{\pi} \ln \sqrt{x^{2}+y^{2}}\right) \\
& \leq \sup _{\partial K}\left(u(x, y)+\frac{1}{\pi} \ln \sqrt{x^{2}+y^{2}}\right)+\sup _{\partial K}\left(-\frac{1}{\pi} \ln \sqrt{x^{2}+y^{2}}\right) \\
& \leq \sup _{B(0,1)}\left(u(x, y)+\frac{1}{\pi} \ln \sqrt{x^{2}+y^{2}}\right)-\frac{1}{\pi} \ln \delta \leq C-\frac{1}{\pi} \ln \delta,
\end{aligned}
$$

where we have used (7) in the last inequality.

Similarly, one gets

$$
\begin{aligned}
\inf _{\partial K} u(x, y) & =\inf _{\partial K}\left(u(x, y)+\frac{1}{\pi} \ln \sqrt{x^{2}+y^{2}}-\frac{1}{\pi} \ln \sqrt{x^{2}+y^{2}}\right) \\
& \geq \inf _{\partial K}\left(u(x, y)+\frac{1}{\pi} \ln \sqrt{x^{2}+y^{2}}\right)-\sup _{\partial K}\left(\frac{1}{\pi} \ln \sqrt{x^{2}+y^{2}}\right) \\
& \geq \inf _{B(0,1)}\left(u(x, y)+\frac{1}{\pi} \ln \sqrt{x^{2}+y^{2}}\right)-\frac{1}{\pi} \ln \delta \\
& \geq-C-\frac{1}{\pi} \ln \delta>0,
\end{aligned}
$$

for all $\delta<e^{-\pi C}=: \delta_{0}(C)=\delta_{0}\left(\mu_{i}\right)$.

Plugging these estimates for the supremum and the infimum into (8), we end up with

$$
\frac{u(x, y)}{C-\frac{1}{\pi} \ln \delta} \leq \mathbf{P}^{(x, y)}(\tau<\infty) \leq \frac{u(x, y)}{-C-\frac{1}{\pi} \ln \delta} .
$$

for $\delta<\delta_{0}\left(\mu_{i}\right)$ and uniformly for $(x, y)$, s.t. $\sqrt{x^{2}+y^{2}} \geq \delta$. This finishes the proof of Case 1 .

General case: $\sigma_{1}, \sigma_{2} \in \mathbf{R}^{+}$.

We indicate the parameter dependence of our stopping time $\tau$ in the following by $\tau_{\left(\mu_{1}, \mu_{2}, \sigma_{1}, \sigma_{2}\right)}^{(x, y, \delta}$, and find

$$
\begin{aligned}
& \tau_{\left(\mu_{1}, \mu_{2}, \sigma_{1}, \sigma_{2}\right)}^{(x, y, \delta)} \\
& :=\inf \left\{t>0 \mid\left(x+\mu_{1} t+\sigma_{1} W_{t}^{(1)}\right)^{2}+\left(y-\mu_{2} \int_{0}^{t} \operatorname{sgn}\left(Y_{s}\right) d s+\sigma_{2} W_{t}^{(2)}\right)^{2}=\delta^{2}\right\} \\
& \quad=\inf \left\{t>0 \mid \sigma_{1}^{2}\left(\frac{x}{\sigma_{1}}+\frac{\mu_{1}}{\sigma_{1}} t+W_{t}^{(1)}\right)^{2}+\sigma_{2}^{2}\left(\frac{y}{\sigma_{2}}-\frac{\mu_{2}}{\sigma_{2}} \int_{0}^{t} \operatorname{sgn}\left(Y_{s}\right) d s+W_{t}^{(2)}\right)^{2}=\delta^{2}\right\}
\end{aligned}
$$




$$
\begin{aligned}
& \leq \inf \left\{t>0 \mid\left(\frac{x}{\sigma_{1}}+\frac{\mu_{1}}{\sigma_{1}} t+W_{t}^{(1)}\right)^{2}+\left(\frac{y}{\sigma_{2}}-\frac{\mu_{2}}{\sigma_{2}} \int_{0}^{t} \operatorname{sgn}\left(Y_{s}\right) d s+W_{t}^{(2)}\right)^{2}=\frac{\delta^{2}}{\min \left(\sigma_{1}^{2}, \sigma_{2}^{2}\right)}\right\} \\
& =\tau_{\left(\mu_{1} / \sigma_{1}, \mu_{2} / \sigma_{2}, 1,1\right)}^{\left(x / \sigma_{1}, y / \sigma_{2}, \delta / \sqrt{\min \left(\sigma_{1}^{2}, \sigma_{2}^{2}\right)}\right)} .
\end{aligned}
$$

Analogously one gets a lower estimate and all together

$$
\tau_{\left(\mu_{1} / \sigma_{1}, \mu_{2} / \sigma_{2}, 1,1\right)}^{\left(x / \sigma_{1}, y / \sigma_{2}, \delta / \sqrt{\left.\max \left(\sigma_{1}^{2}, \sigma_{2}^{2}\right)\right)}\right.} \leq \tau_{\left(\mu_{1}, \mu_{2}, \sigma_{1}, \sigma_{2}\right)}^{(x, y)} \leq \tau_{\left(\mu_{1} / \sigma_{1}, \mu_{2} / \sigma_{2}, 1,1\right)}^{\left(x / \sigma_{1}, y / \sigma_{2}, \delta / \sqrt{\min \left(\sigma_{1}^{2}, \sigma_{2}^{2}\right)}\right)} .
$$

Using Case 1, one gets

$$
\mathbf{P}\left(\tau_{\left(\mu_{1} / \sigma_{1}, \mu_{2} / \sigma_{2}, 1,1\right)}^{\left(x / \sigma_{1}, y / \sigma_{2}, \delta / \sqrt{\max \left(\sigma_{1}^{2}, \sigma_{2}^{2}\right)}\right)}<\infty\right) \sim \pi u\left(\frac{x}{\sigma_{1}}, \frac{y}{\sigma_{2}} ; \frac{\mu_{1}}{\sigma_{1}}, \frac{\mu_{2}}{\sigma_{2}}\right) \frac{1}{\left(-\ln \frac{\delta}{\sqrt{\max \left(\sigma_{1}^{2}, \sigma_{2}^{2}\right)}}\right)}
$$

uniformly for $\frac{x^{2}}{\sigma_{1}^{2}}+\frac{y^{2}}{\sigma_{2}^{2}} \geq \frac{\delta^{2}}{\max \left(\sigma_{1}^{2}, \sigma_{2}^{2}\right)}$, hence uniformly for $x^{2}+y^{2} \geq \delta^{2}$

An analogous calculation with the maximum replaced by the minimum, gives the same asymptotic relation (with the minimum replacing the maximum), and now uniformly on $x^{2}+y^{2} \geq \delta^{2} \frac{\max \left(\sigma_{1}^{2}, \sigma_{2}^{2}\right)}{\min \left(\sigma_{1}^{2}, \sigma_{2}^{2}\right)}$. Finally, as

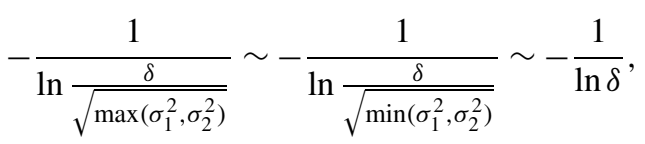

the theorem is proved.

\section{A stochastic optimal control problem}

As announced in the introduction, we show in this section that the process $Z_{t}$, which we are considering, solves a certain stochastic control problem, namely the following: Let $Z_{t}^{u}:=\left(X_{t}^{u}, Y_{t}^{u}\right)$, where

$$
\begin{aligned}
& X_{t}^{u}=x+\mu_{1} t+W_{t}^{(1)}, \\
& Y_{t}^{u}=y+\mu_{2} \int_{0}^{t} u_{s} d s+W_{t}^{(2)},
\end{aligned}
$$

where $u_{s}$ is a Borel measurable function of $\left(s, Z_{s}\right)$, with $\left|u\left(s, Z_{s}\right)\right| \leq 1$ and $\mu_{i}>0$. It is well known, see [15], that (12) has a strong solution. With the previously defined notation, we consider the problem

$$
\mathbf{P}(\tau<\infty) \rightarrow \max
$$

that is, we allow the controller to influence only the drift in one of two orthogonal directions, and we want to maximize the probability that the process hits the small circle. Intuitively, it is rather clear that the process, we have considered in this paper, solves this problem. Nevertheless, we give a short proof, namely with the notation $u^{*}$ for the optimal strategy we show the following

Proposition 3.1. For the problem (13), the strategy $u^{*}:=-\operatorname{sgn}\left(Y_{t}\right)$ is optimal. 
Proof. We want to maximize

$$
\mathbf{P}\left(X_{t}^{2}+Y_{t}^{2}=\delta^{2} \text { for some } t\right),
$$

which is the same as to maximize

$$
\mathbf{E}\left[\mathbf{P}\left(X_{t}^{2}+Y_{t}^{2}=\delta^{2} \text { for some } t \mid X_{t}, t \in[0, \infty)\right)\right]
$$

Now, if a strategy $u_{t}$ is optimal for the problem

$$
\mathbf{P}\left(Y_{t}^{2}=\delta^{2}-f^{2}(t) \text { for some } t\right) \rightarrow \max ,
$$

for an arbitrary continuous function $f(t)$, with $f(0)=x$, then it is clearly optimal for problem (14). The fact that the strategy given in the formulation of the proposition fulfills this, follows directly from [8], Theorem 2.1 (iii), if we take into account the following points.

First of all, although the Ikeda-Watanabe result is formulated for controls, fulfilling $\left|u_{s}\right| \leq 1$, it holds as well for $\left|u_{s}\right| \leq r$, for some positive constant $r$. This can be seen by a time scaling argument. Moreover, as in their application of Theorem 2.1, namely the corollary following this theorem, our optimization problem concerns only properties depending on the law of the process (certain expectations in their case).

\section{Some degenerate cases and numerical examples}

It is instructive to study the cases, where one or both volatilities $\sigma_{i}$ vanish. We shall see that the magnitude of the hitting probability, which was $O(-1 / \ln \delta)$ in the non-degenerate case, changes. We are satisfied in these degenerate cases with the determination of the the correct order for the hitting probability, and we do not care about the $(x, y)$-dependence of its coefficient function. For convenience, we set the remaining volatility equal to one. We start with the easier case.

Case I: $\sigma_{1}=1, \sigma_{2}=0$.

In this case, we have the evolution equations

$$
\begin{aligned}
& X_{t}=x+\mu_{1} t+W_{t}^{(1)}, \\
& Y_{t}=y-\mu_{2} \int_{0}^{t} \operatorname{sgn}\left(Y_{s}\right) d s .
\end{aligned}
$$

We solve the second one explicitly and get

$$
Y_{t}= \begin{cases}y-\operatorname{sgn}(y) \mu_{2} t, & t \leq \frac{|y|}{\mu_{2}}=: t^{*}, \\ 0, & t \geq t^{*}\end{cases}
$$

Clearly, we have, for $\delta \rightarrow 0$,

$$
\begin{aligned}
\mathbf{P}(\tau<\infty) & \sim \mathbf{P}\left(X_{t^{*}}<0\right)+\gamma=\mathbf{P}\left(x+\mu_{1} t^{*}+W_{t^{*}}^{(1)}<0\right)+\gamma \\
& =\Phi\left(-x \sqrt{\frac{\mu_{2}}{|y|}}-\mu_{1} \sqrt{\frac{|y|}{\mu_{2}}}\right)+\gamma
\end{aligned}
$$


where

$$
\gamma:=\int_{0}^{\infty} \mathbf{P}\left(\inf _{t>0}\left(z+\mu_{1} t+W_{t}^{(1)}\right)<0\right) \mathbf{P}\left(X_{t^{*}} \in d z\right) .
$$

We see that the hitting probability is much larger than in the non-degenerate case, namely it has order $O(1)$. This fits nicely with the intuition, since the process in the controlled direction does not face any stochastic perturbations.

Case II: $\sigma_{1}=0, \sigma_{2}=1$.

Case II.1: $x<0$

We first note that we have in this case $x<-\delta$, for $\delta$ small enough, since we stick here to our assumption that $(x, y)$ should not depend on $\delta$. Moreover, we assume, w.l.o.g., $y \geq 0$.

Let us denote by $\tau_{1}$ the hitting time for a square with the origin as center and with side length of $2 \delta$. Obviously, we have $\tau_{1}<\tau$, a.s., hence

$$
\mathbf{P}(\tau<\infty)<\mathbf{P}\left(\tau_{1}<\infty\right) .
$$

Our evolution equations become

$$
\begin{aligned}
& X_{t}=x+\mu_{1} t \\
& Y_{t}=y-\mu_{2} \int_{0}^{t} \operatorname{sgn}\left(Y_{s}\right) d s+W_{t}^{(2)} .
\end{aligned}
$$

We now note that, for $t^{*}:=\frac{-\delta-x}{\mu_{1}}$, we have $X_{t^{*}}=-\delta$, and define finally the sets $A_{1}:=\{y \mid y>\delta\}$, $A_{2}:=\{y \mid y<-\delta\}$ and $A_{3}:=\{y|| y \mid \leq \delta\}$. With these definitions, we calculate

$$
\begin{aligned}
\mathbf{P}\left(\tau_{1}<\infty\right)= & \int_{-\infty}^{\infty} \mathbf{P}\left(\tau_{1}<\infty, Y_{t^{*}} \in d w\right)=\int_{A_{1}} \mathbf{P}\left(\tau_{1}<\infty \mid Y_{t^{*}}=w\right) \mathbf{P}\left(Y_{t^{*}} \in d w\right) \\
& +\int_{A_{2}} \mathbf{P}\left(\tau_{1}<\infty \mid Y_{t^{*}}=w\right) \mathbf{P}\left(Y_{t^{*}} \in d w\right)+\int_{A_{3}} \mathbf{P}\left(\tau_{1}<\infty \mid Y_{t^{*}}=w\right) \mathbf{P}\left(Y_{t^{*}} \in d w\right) \\
=: & J_{1}+J_{2}+J_{3} .
\end{aligned}
$$

Obviously, we have $J_{3}=\mathbf{P}\left(\left|Y_{t^{*}}\right| \leq \delta\right)$, and for $J_{2}$ we find by symmetry

$$
\begin{aligned}
J_{2} & =\int_{A_{2}} \mathbf{P}\left(\tau_{1}<\infty \mid Y_{t^{*}}=-w\right) \mathbf{P}\left(Y_{t^{*}} \in d w\right) \\
& =\int_{A_{1}} \mathbf{P}\left(\tau_{1}<\infty \mid Y_{t^{*}}=z\right) \mathbf{P}\left(Y_{t^{*}} \in-d z\right) \\
& =\int_{A_{1}} \mathbf{P}\left(\tau_{1}<\infty \mid Y_{t^{*}}=w\right) \mathbf{P}\left(-Y_{t^{*}} \in d w\right) .
\end{aligned}
$$

Hence, we get

$$
\mathbf{P}\left(\tau_{1}<\infty\right)=\int_{A_{1}} \mathbf{P}\left(\tau_{1}<\infty \mid Y_{t^{*}}=w\right) \mathbf{P}\left(\left|Y_{t^{*}}\right| \in d w\right)+\mathbf{P}\left(\left|Y_{t^{*}}\right| \leq \delta\right) .
$$

The process $\left|Y_{t}\right|$ is a realization of the reflected Brownian motion with negative drift, see [6], and the distribution of $\left|Y_{t^{*}}\right|$ is well known, see [11]. Let $F^{R}$ be the distribution function of $\left|Y_{t^{*}}\right|$. 
On the other hand, the quantity $\mathbf{P}\left(\tau_{1}<\infty \mid Y_{t^{*}}=w\right)$, for $w \in A_{1}$, is explicitly known: [3], 2.1.1.4, p. 197 provides

$$
\begin{aligned}
\mathbf{P}\left(\tau_{1}<\infty \mid Y_{t^{*}}=w\right)= & \frac{1}{2} \operatorname{erfc}\left(\frac{w-\delta}{2 \sqrt{\delta}}-\mu_{2} \sqrt{\delta}\right) \\
& +\frac{1}{2} e^{2 \mu_{2}(w-\delta)} \operatorname{erfc}\left(\frac{w-\delta}{2 \sqrt{\delta}}+\mu_{2} \sqrt{\delta}\right) \\
= & : g(w) .
\end{aligned}
$$

(17) and (18) yield

$$
\mathbf{P}\left(\tau_{1}<\infty\right)=\int_{\delta}^{\infty} g(w) d F^{R}(w)+\mathbf{P}\left(\left|Y_{t^{*}}\right| \leq \delta\right),
$$

hence,

$$
\mathbf{P}\left(\tau_{1}<\infty\right) \leq \sup _{w \geq \delta} f^{R}(w) \int_{\delta}^{\infty} g(w) d w+\mathbf{P}\left(\left|Y_{t^{*}}\right| \leq \delta\right),
$$

where $f^{R}$ denotes the density corresponding to $F^{R}$, and the $g$-integral can be calculated explicitly, giving

$$
\int_{\delta}^{\infty} g(w) d w=\mu_{2} \delta \operatorname{erfc}\left(-\mu_{2} \sqrt{\delta}\right)+\sqrt{\frac{\delta}{\pi}} e^{-\mu_{2}^{2} \delta}+\frac{1-\operatorname{erfc}\left(\mu_{2} \sqrt{\delta}\right)}{2 \mu_{2}} .
$$

Clearly, this gives

$$
\int_{\delta}^{\infty} g(w) d w \sim \text { const. }\left(\mu_{i}\right) \sqrt{\delta}
$$

for some positive constant.

On the other hand, we find for $f^{R}$, by using [11], Section 4.2, that

$$
\begin{aligned}
f^{R}(w)= & \phi\left(\frac{w-y+\mu_{2} t^{*}}{\sqrt{t^{*}}}\right) \frac{1}{\sqrt{t^{*}}}+2 \mu_{2} e^{-2 \mu_{2} w} \Phi\left(\frac{-w-y+\mu_{2} t^{*}}{\sqrt{t^{*}}}\right) \\
& +e^{-2 \mu_{2} w} \phi\left(\frac{-w-y+\mu_{2} t^{*}}{\sqrt{t^{*}}}\right) \frac{1}{\sqrt{t^{*}}},
\end{aligned}
$$

where $\phi$ denotes the standard normal density. Since $t^{*} \geq$ const. $\left(\mu_{i}, x\right)>0$ holds, we have

$$
f^{R}(w) \leq \text { const. }\left(x, \mu_{i}\right)
$$

and

$$
\mathbf{P}\left(\left|Y_{t^{*}}\right| \leq \delta\right) \leq \text { const. }\left(x, \mu_{i}\right) \delta,
$$

for some positive constants. Now, (16),(20),(22),(24) and (25) yield

$$
\mathbf{P}(\tau<\infty) \leq U,
$$

for some function $U$, with $U \sim$ const. $\left(\mu_{i}, x\right) \sqrt{\delta}$. 
To find a lower estimate, define the stopping time $\tau_{2}$ as the hitting time for the square $[-\delta / 2, \delta / 2]^{2}$, which implies $\tau_{2} \geq \tau$. Completely analogously as for (19) one finds

$$
\mathbf{P}\left(\tau_{2}<\infty\right)=\int_{\delta / 2}^{\infty} g(w) d F^{R}(w)+\mathbf{P}\left(\left|Y_{t^{*}}\right| \leq \delta / 2\right),
$$

thereby replacing $\delta$ by $\delta / 2$ at the appropriate places in the definitions of $g$ and $F^{R}$. From (23) we have $\inf _{w \in[\delta / 2,1]} f^{R}(w) \geq$ const. $\left(x, y, \mu_{i}\right)>0$, implying

$$
\begin{aligned}
\mathbf{P}\left(\tau_{2}<\infty\right) & \geq \inf _{w \in[\delta / 2,1]} f^{R}(w) \int_{\delta / 2}^{1} g(w) d w \\
& \geq \text { const. }\left(x, y, \mu_{i}\right)\left(\int_{\delta / 2}^{\infty} g(w) d w-\int_{1}^{\infty} g(w) d w\right) .
\end{aligned}
$$

The last term in the bracket can easily shown to be smaller than $e^{-\frac{\text { const. }}{\delta}}$, for some positive constant. Hence, we have

$$
\mathbf{P}(\tau<\infty) \geq \mathbf{P}\left(\tau_{2}<\infty\right) \geq V,
$$

for some function $V$, with $V \sim$ const. $\left(\mu_{i}, x, y\right) \sqrt{\delta}$. (26) and (28) yield

$$
\mathbf{P}(\tau<\infty) \approx \sqrt{\delta}
$$

where now, as announced at the beginning of this section, the constants in the definition of $\approx$ may depend on $(x, y)$.

Case II.2: $x=0$ (hence $y \geq 1$ ).

Here one finds easily

$$
\mathbf{P}(\tau<\infty) \leq e^{-\frac{c}{\delta}},
$$

for some positive constant $c$.

Case II.3: $x>0$.

Obviously,

$$
\mathbf{P}(\tau<\infty)=0
$$

holds, when $\delta \leq x$.

One observes that in the case, where the volatility in the controllable direction "strongly dominates" the volatility in the non controllable direction, a much smaller order for the hitting probability appears.

Let us summarize the findings of this section in the following lemma, and let us note again that $\approx$ now incorporates $(x, y)$ dependent constants.

Lemma 4.1. The orders of the hitting probabilities in the considered degenerate cases are as follows:

$$
\begin{aligned}
& \sigma_{1}=1, \sigma_{2}=0, x \in \mathbf{R} \quad \Rightarrow \quad \mathbf{P}(\tau<\infty) \approx 1, \\
& \sigma_{1}=0, \sigma_{2}=1, x<0 \quad \Rightarrow \quad \mathbf{P}(\tau<\infty) \approx \sqrt{\delta}, \\
& \sigma_{1}=0, \sigma_{2}=1, x=0 \quad \Rightarrow \quad \mathbf{P}(\tau<\infty) \leq e^{-\frac{c}{\delta}}, \\
& \sigma_{1}=0, \sigma_{2}=1, x>0 \quad \Rightarrow \quad \mathbf{P}(\tau<\infty)=0,
\end{aligned}
$$

for some positive constant $c$, depending on $\left(x, y, \mu_{i}\right)$. 


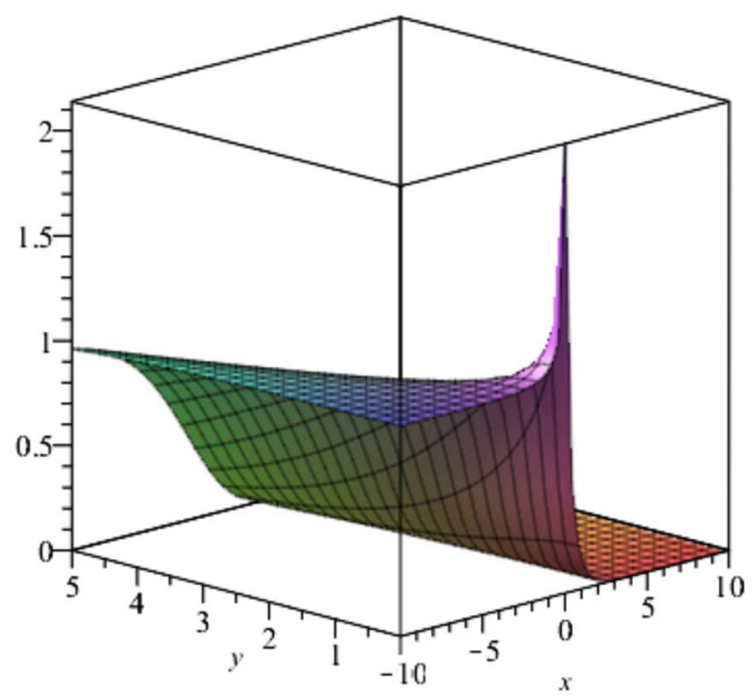

Figure 1. Plot of the function $u(x, y)$ for the parameters $\mu_{1}=\mu_{2}=\sigma_{1}=1, \sigma_{2}=1$.

We close this paper with two numerical examples (see Figures 1 and 2), for which we plot the function $u(x, y)$ appearing in Theorem 2.1 for the indicated parameter values. One observes a smaller function $u(x, y)$ for the case where the second volatility is higher, which is in accordance with intuition.

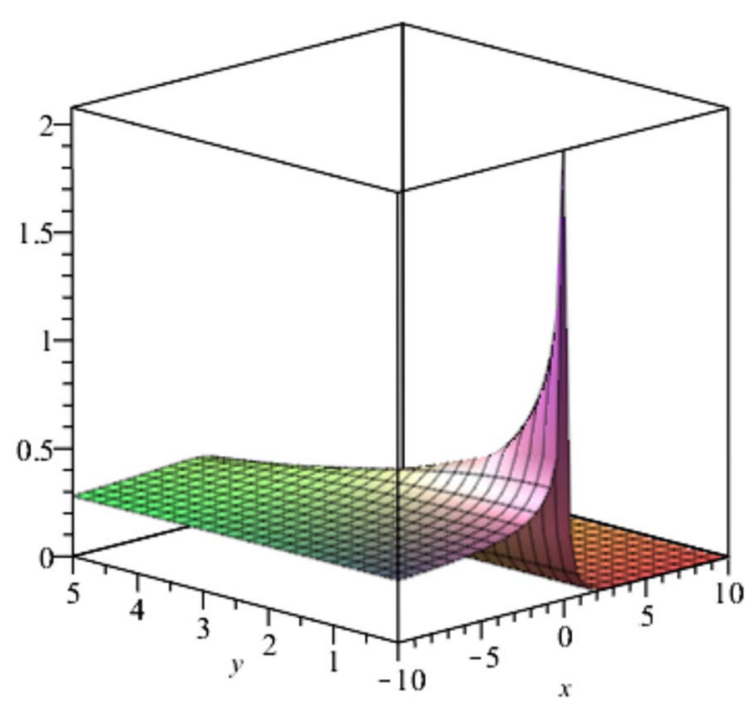

Figure 2. Plot of the function $u(x, y)$ for the parameters $\mu_{1}=\mu_{2}=\sigma_{1}=1, \sigma_{2}=4$. 


\section{Appendix}

Lemma A.1. For the function $u(x, y)$ defined in (4) we have

$$
u(x, y)=\mathbf{E}^{(x, y)}\left[\mathbf{1}_{\{\tau<\infty\}} u\left(X_{\tau}, Y_{\tau}\right)\right]
$$

Proof. We first define, by a slight abuse of notation, the potential kernel with pole at $(r, s)$ as $u(x, y ; r, s)$. Then we use the following result, the reader can find, for example, in [5] (5.6'), (5.4) and (2.27):

If

$$
f(x, y)=\mathbf{E}^{(x, y)}\left[\int_{0}^{\infty} h\left(X_{t}, Y_{t}\right) d t\right]=\int_{\mathbf{R}^{2}} u(x, y ; r, s) h(r, s) d r d s
$$

holds, then we have for any stopping time $\tau$,

$$
\mathbf{E}^{(x, y)}\left[f\left(X_{\tau}, Y_{\tau}\right)\right]=f(x, y)-\mathbf{E}^{(x, y)}\left[\int_{0}^{\tau} h\left(X_{t}, Y_{t}\right) d t\right] .
$$

If $\tau$ is the first hitting time of a ball, and $h$ is zero outside this ball, then we simply have

$$
\mathbf{E}^{(x, y)}\left[f\left(X_{\tau}, Y_{\tau}\right)\right]=f(x, y) .
$$

We now use an approximating sequence for the Dirac Delta as $h$, say $h_{n}(r, s):=n \mathbf{1}_{B_{n}}(r, s)$, with $B_{n}:=B(0,1 / \sqrt{\pi n})$. Hence, we get

$$
\mathbf{E}^{(x, y)}\left[\int_{\mathbf{R}^{2}} u\left(X_{\tau}, Y_{\tau} ; r, s\right) n \mathbf{1}_{B_{n}}(r, s) d r d s\right]=\int_{\mathbf{R}^{2}} u(x, y ; r, s) n \mathbf{1}_{B_{n}}(r, s) d r d s .
$$

We now let $n \rightarrow \infty$ in the previous equation and start with the right hand side. If, for fixed $(x, y)$, the function $u(x, y ; r, s)$ is continuous at $(r, s)=(0,0)$, the limit would be $u(x, y ; 0,0)$. This continuity follows from the explicit form of $u$ given by (see [3], Section 15, Appendix 1, for $p_{t}^{Y}$ ),

$$
\begin{aligned}
u(x, y ; r, s)= & \int_{0}^{\infty} p_{t}^{X}(x, r) p_{t}^{Y}(y, s) d t \\
= & \int_{0}^{\infty} \frac{1}{\sqrt{2 \pi t}} \exp \left(-\frac{\left(r-x-\mu_{1} t\right)^{2}}{2 t}\right)\left[\frac{1}{\sqrt{2 \pi t}} \exp \left(-\mu_{2}(|y|+|s|)-\frac{\mu_{2}^{2}}{2} t-\frac{(y-s)^{2}}{2 t}\right)\right. \\
& \left.+\frac{\mu_{2}}{2} \operatorname{erfc}\left(\frac{|y|+|s|-\mu_{2} t}{\sqrt{2 t}}\right)\right] d t
\end{aligned}
$$

the fact that $(x, y)$ is by assumption bounded away from zero, and the dominated convergence theorem.

For the left-hand side of (33) we find

$$
\begin{aligned}
& \mathbf{E}^{(x, y)}\left[\int_{\mathbf{R}^{2}} u\left(X_{\tau}, Y_{\tau} ; r, s\right) n \mathbf{1}_{B_{n}}(r, s) d r d s\right] \\
& =\mathbf{E}^{(x, y)}\left[\mathbf{1}_{\{\tau<\infty\}} \int_{\mathbf{R}^{2}} u\left(X_{\tau}, Y_{\tau} ; r, s\right) n \mathbf{1}_{B_{n}}(r, s) d r d s\right]
\end{aligned}
$$




$$
\begin{aligned}
& +\mathbf{E}^{(x, y)}\left[\mathbf{1}_{\{\tau=\infty\}} \int_{\mathbf{R}^{2}} u\left(X_{\tau}, Y_{\tau} ; r, s\right) n \mathbf{1}_{B_{n}}(r, s) d r d s\right] \\
= & \mathbf{E}^{(x, y)}\left[\mathbf{1}_{\{\tau<\infty\}} \int_{\mathbf{R}^{2}} u\left(X_{\tau}, Y_{\tau} ; r, s\right) n \mathbf{1}_{B_{n}}(r, s) d r d s\right],
\end{aligned}
$$

where the last equality follows from the fact that $u(x, y ; r, s) \rightarrow 0$, for $x \rightarrow \infty$, uniformly in $y, r, s$ (see the explicit expression for $u$ above), and the fact that $X_{t}$ tends to $\infty$ on $\{\tau=\infty\}$, a.s.. Analogously to the right-hand side of (33), one shows that this converges for $n \rightarrow \infty$ to $\mathbf{E}^{(x, y)}\left[\mathbf{1}_{\{\tau<\infty\}} u\left(X_{\tau}, Y_{\tau} ; 0,0\right)\right]$. All together, we find

$$
\mathbf{E}^{(x, y)}\left[\mathbf{1}_{\{\tau<\infty\}} u\left(X_{\tau}, Y_{\tau}\right)\right]=u(x, y),
$$

proving our lemma.

\section{Acknowledgements}

I want to thank two anonymous referees for carefully reading my article and suggestions, which lead to a considerable simplification of the paper.

Support by the "Austrian Science Foundation" (Fonds zur Förderung der Wissen-schaftlichen Forschung), Project nr. P30864-N35, is gratefully acknowledged.

\section{References}

[1] Asmussen, S. and Albrecher, H. (2010). Ruin Probabilities, 2nd ed. Advanced Series on Statistical Science \& Applied Probability 14. Hackensack, NJ: World Scientific Co. Pte. Ltd. MR2766220 https://doi.org/10. 1142/9789814282536

[2] Beneš, V.E., Shepp, L.A. and Witsenhausen, H.S. (1980/81). Some solvable stochastic control problems. Stochastics 4 39-83. MR0587428 https://doi.org/10.1080/17442508008833156

[3] Borodin, A.N. and Salminen, P. (2002). Handbook of Brownian Motion-Facts and Formulae, 2nd ed. Probability and Its Applications. Basel: Birkhäuser. MR1912205 https://doi.org/10.1007/978-3-0348-8163-0

[4] Collamore, J.F. (1996). Hitting probabilities and large deviations. Ann. Probab. 24 2065-2078. MR1415241 https://doi.org/10.1214/aop/1041903218

[5] Dynkin, E.B. (1965). Markov Processes. Vol 1. Berlin: Springer.

[6] Graversen, S.E. and Shiryaev, A.N. (2000). An extension of P. Lévy's distributional properties to the case of a Brownian motion with drift. Bernoulli 6 615-620. MR1777686 https://doi.org/10.2307/3318509

[7] Grzywny, T. and Kwaśnicki, M. (2018). Potential kernels, probabilities of hitting a ball, harmonic functions and the boundary Harnack inequality for unimodal Lévy processes. Stochastic Process. Appl. 128 1-38. MR3729529 https://doi.org/10.1016/j.spa.2017.04.004

[8] Ikeda, N. and Watanabe, S. (1977). A comparison theorem for solutions of stochastic differential equations and its applications. Osaka J. Math. 14 619-633. MR0471082

[9] Karatzas, I. and Shreve, S.E. (1991). Brownian Motion and Stochastic Calculus, 2nd ed. Graduate Texts in Mathematics 113. New York: Springer. MR1121940 https://doi.org/10.1007/978-1-4612-0949-2

[10] Kolmogorov, A.N., Miščenko, E.F. and Pontrjagin, L.S. (1962). A probability problem of optimal control. Dokl. Akad. Nauk SSSR 145 993-995. MR0183574

[11] Linetsky, V. (2005). On the transition densities for reflected diffusions. Adv. in Appl. Probab. 37 435-460. MR2144561 https://doi.org/10.1239/aap/1118858633

[12] Lundberg, F. (1903). Approximerad Framställning Av Sannolikehetsfunktionen, Återförsäkering Av Kollektivrisker. Uppsala: Almqvist and Wiksell.

[13] Nikol'skiı̌, M.S. (1964). The probability of a Markov point hitting a planar region of small diameter. Theory Probab. Appl. 9 352-357. MR0163359 
[14] Revuz, D. and Yor, M. (1999). Continuous Martingales and Brownian Motion, 3rd ed. Grundlehren der Mathematischen Wissenschaften [Fundamental Principles of Mathematical Sciences] 293. Berlin: Springer. MR1725357 https://doi.org/10.1007/978-3-662-06400-9

[15] Veretennikov, A.Yu. (1982). Criteria for the existence of a strong solution of a stochastic equation. Teor. Veroyatn. Primen. 27 417-424. MR0673915

[16] Wendel, J.G. (1980). Hitting spheres with Brownian motion. Ann. Probab. 8 164-169. MR0556423

[17] Yin, C. (1999). The joint distribution of the hitting time and place to a sphere or spherical shell for Brownian motion with drift. Statist. Probab. Lett. 42 367-373. MR1707182 https://doi.org/10.1016/S0167-7152(98) 00231-4

[18] Zvonkin, A.K. (1974). A transformation of the phase space of a diffusion process that will remove the drift. Mat. Sb. (N.S.) 22 129-149. MR0336813

Received May 2019 and revised August 2020 